\title{
Evidence that the Amyloid beta Precursor Protein-intracellular domain lowers the stress threshold of neurons and has a "regulated" transcriptional role
} Luca Giliberto ${ }^{1,4}$, Dawang Zhou1 ${ }^{1}$ Richard Weldon¹, Elena Tamagno², Pasquale De Luca ${ }^{3}$, Massimo Tabaton ${ }^{4}$ and Luciano D'Adamio*1,5

Address: ${ }^{1}$ Department of Microbiology and Immunology, Albert Einstein College of Medicine, 1300 Morris Park Ave, Bronx, NY 10461, USA, ${ }^{2}$ Department of Experimental Medicine and Oncology, General Pathology Section, University of Turin, Turin, Italy, ${ }^{3} \mathrm{SZN}-\mathrm{BioGeM}$, Ariano Irpino, Italy, ${ }^{4}$ Department of Neurosciences, Ophthalmology, and Genetics, University of Genova, Genova, Italy and ${ }^{5}$ Dipartimento di Biochimica e Biotecnologie Mediche, Università di Napoli Federico II, Naples, Italy

Email: Luca Giliberto - luca.giliberto@unige.it; Dawang Zhou - dwzhoulj@yahoo.com; Richard Weldon - rweldon@aecom.yu.edu; Elena Tamagno - elena.tamagno@unito.it; Pasquale De Luca - deluca@biogem.it; Massimo Tabaton - Massimtabaton@neurologia.unige.it; Luciano D'Adamio* - ldadamio@aecom.yu.edu

* Corresponding author

Published: 2 September 2008

Molecular Neurodegeneration 2008, 3:12 doi:10.1186/1750-1326-3-12
Received: 19 July 2008

Accepted: 2 September 2008

This article is available from: http://www.molecularneurodegeneration.com/content/3/1/12

(c) 2008 Giliberto et al; licensee BioMed Central Ltd.

This is an Open Access article distributed under the terms of the Creative Commons Attribution License (http://creativecommons.org/licenses/by/2.0), which permits unrestricted use, distribution, and reproduction in any medium, provided the original work is properly cited.

\begin{abstract}
Background: Regulated intramembrane proteolysis of the $\beta$-amyloid precursor protein by the $\gamma$ secretase yields two peptides. One, amyloid- $\beta$, is the major component of the amyloid plaques found in Alzheimer's disease patients. The other, APP IntraCellular Domain, has been involved in regulation of apoptosis, calcium flux and gene transcription. To date, a few potential target genes transcriptionally controlled by AID, alone or complexed with Fe65/Tip60, have been described. Although the reports are controversial: these include KAII, Neprilysin, p53, EGFR, LRP and APP itself. Furthermore, $p 53$ has been implicated in AID mediated susceptibility to apoptosis. To extend these findings, and assess their in vivo relevance, we have analyzed the expression of the putative target genes and of the total brain basal transriptoma in transgenic mice expressing AID in the forebrain. Also, we have studied the susceptibility of primary neurons from such mice to stress and proapoptotic agents.
\end{abstract}

Results: We found that AID-target genes and the mouse brain basal transcriptoma are not influenced by transgenic expression of AID alone, in the absence of Fe65 over-expression. Also, experiments conducted on primary neurons from AID transgenic mice, suggest a role for AID in sensitizing these cells to toxic stimuli. Overall, these findings hint that a role for AID, in regulating gene transcription, could be induced by yet undefined, and possibly stressful, stimuli in vivo.

Conclusion: Overall, these data suggest that the release of the APP intracellular domain may modulate the sensitivity of neuronal cells to toxic stimuli, and that a transcriptional role of AID could be inscribed in signaling pathways thatare not activated in basal conditions. 


\section{Background}

Processing of Amyloid Precursor Protein (APP) by $\beta$ - and $\gamma$-secretase produces $A \beta$ peptides and APP IntraCellular Domain $[1,2]$, the former being the major component of AD amyloid plaques. Recent evidence indicates that AID is a biologically active intracellular peptide. Initial findings indicated that AID could sensitize cells to apoptotic stimuli [3]. Subsequent studies have suggested a role of AID in calcium release from endoplasmic reticulum stores [4]and in gene transcription [5]. The putative transcriptional role of AID has attracted most of the attention because of the functional parallel with Notch signaling, another $\gamma$-secretase substrate. In the case of Notch, $\gamma$ processing releases NICD that, in the nucleus, binds transcription factors and activates transcription of specific gene targets $[6,7]$. For APP, similar models have been suggested, where AID travels to the nucleus bound to Fe65 and Tip60 to activate transcription of target genes [5]; furthermore, Fe65 would also boost AID generation [8]. The evidence that AID, Fe65 and Tip 60 can all be found on the KAI1 [9] and Neprilysin (NEP) [10] promoters supports this model. AID gene targets that have been described so far include KAI1 [9,11], GSK3 $\beta[11,12]$, NEP [10], EGFR [13], LRP [14] and APP itself [15], and genes involved in cell cycle control [16] and in tumorigenesis [13]. A genome-wide approach to AID-mediated gene transcription has shown a possible effect of AID in regulating the expression of proteins related to cytoskeletal organization [17] but failed to confirm previous target genes, as have other studies $[18,19]$. Given this ambiguity in results, we have reexamined the role of AID in transcription and apoptosis in vivo studying AID-transgenic (AIDtg) mice. We have found that AID does not univocally regulate the basal expression of APP, NEP, KAI1 and $p 53$ in vivo in the mouse brain and that the brain transcriptome of AIDtg and littermate mice are identical. Altogether, these findings suggest that a transcriptional role for AID could be inducible. Nonetheless, toxicity tests performed on forebrain primary cortical neurons from AIDtg mice show that AID has the potential to sensitize neurons to toxic stimuli, possibly via a p53-dependent pathway $[20,21]$.

\section{Materials and methods Construction of the transgenic plasmid}

The cDNA sequence corresponding to human AID 50, 57 or 59 was subcloned into BamHI-XhoI sites of pHY12 vector, which bears SV40 polyA signal. A NotI-NotI fragment, comprising the transgene, was then cloned into the pNN vector, downstream of the 8-kb CaMKII $\alpha$ promoter. The whole plasmid was then linearized with SalI, run on agarose gel, purified and injected into oocytes of FVB mice that were than implanted in pseudo pregnant $\mathrm{C} 57 \mathrm{BL} / 6$ mice.

\section{Mice breeding and handling}

Mice were maintained on a FVB background and handled according to the Ethical Guidelines for Treatment of Laboratory Animals of Albert Einstein College of Medicine. The procedures were described and approved in animal protocol number 20040707.

\section{Mice Genotyping}

Genomic DNA was extracted and purified from mice tails with DNeasy Tissue Kit (Qiagen), according to the manufacturer's protocol. PCR was conducted using Taq PCR Core Kit (Qiagen) and a Touchdown PCR protocol, starting at $60^{\circ} \mathrm{C}$. Primers were constructed on the pNN (fw) and $\mathrm{pHY}$ (rev) vectors used for cloning, as follows: fw: 5'CGAGTGGCCCCTAGTTC-3', rev: 5'-CACTGCATTCTAGTTGTGGTTTG-3'. Internal control primers for $\beta$ Actin are as follows: fw: 5'-ACCCACACTGTGCCCATCTA3'; rev: 5'-CGGAACCGCTACTTGCC-3'. PCR products were run on a $1.5 \%$ TBE agarose gel with $0.05 \%$ Ethidium Bromide.

\section{Mouse Brain Dissection}

Brains were dissected from sacrificed mice using a 3diopter magnification lens, in ice-cold, RNase, DNase free $1 \times$ PBS (Sigma) made in DEPC double distilled water. One hemisphere, for protein extraction, was shock frozen in liquid nitrogen and stored at $-80^{\circ} \mathrm{C}$, the other hemisphere was processed for RNA extraction as described. Forebrains only were utilized.

\section{Primary Neuronal Cultures}

Culture plates were coated with $15 \mu \mathrm{g} / \mathrm{mL}$ Poly-L-Ornithine (Low Molecular Weight, Sigma) for 45 minutes at room temperature. Poly-L-Ornithine was the aspirated and wells were soaked with $4 \mu \mathrm{g} / \mathrm{mL}$ mouse Laminin (Invitrogen), for 12-16 hours in a cell culture incubator at $37^{\circ} \mathrm{C}, 95 \%$ humidity and 5\% CO2. Eight weeks old FVB female mice were bred with age matched male mice for 3 days. Pregnancy was ascertained according to vaginal plug and weight gain of the females. Females were sacrificed by cervical dislocation, after sedation with isoflurane, at 17.5 days of gestation. Foetuses were processed separately, in order to obtain pure transgenic cultures. Genotyping was carried out as described, by isolating tail DNA. Forebrains were dissected in ice cold HBSS (Invitrogen) $+0.5 \% \mathrm{w} / \mathrm{v}$ D-Glucose (Sigma) and $25 \mathrm{mM}$ Hepes (Invitrogen), under a dissection microscope (Zoom 2000, Leica). Dissociation was carried out in ice cold dissection medium plus $0.01 \% \mathrm{w} / \mathrm{v}$ Papain (Worthington), $0.1 \% \mathrm{w} / \mathrm{v}$ Dispase (Roche) and $0.01 \% \mathrm{w} / \mathrm{v}$ DNase (Worthington), first by means of sterile razor blades, then by serial pipetting with flamed sterile glass Pasteur pipettes, and incubation at $37^{\circ} \mathrm{C}$ twice for 15 minutes. Cells were then spun down at $220 \mathrm{~g}$ for $5^{\prime}$ at $4^{\circ} \mathrm{C}$, resuspended in Neurobasal Medium with 2\% B27, $1 \mathrm{mM}$ Na Pyruvate, 100 units/ml penicillin, 
$100 \mu \mathrm{g} / \mathrm{ml}$ streptomycin, $2 \mathrm{mM}$ Glutamax (all from Invitrogen), filtered through a $40 \mu \mathrm{m}$ cell strainer (Fisher), counted and plated on coated 6 well plates at a density of about 750.000 cells/well. Culture medium was completely replaced after 16-20 hours, and new medium (30\% of starting volume) was added every 3 days until needed. mRNA harvest was performed at 14 and 9 DIV. Also, at 9 DIV, neurons were treated for 3 hours with 500 $\mu \mathrm{M} \mathrm{H} 2 \mathrm{O} 2$ in culture medium devoid of Na-Pyruvate, and for 16 hours with either $700 \mu \mathrm{M}$ Kainic Acid (Sigma), 7 $\mathrm{pg} / \mu \mathrm{L}$ FAS Ligand (Upstate), $1 \mu \mathrm{M}$ Staurosporine (Sigma), $1 \mu \mathrm{M}$ A $\beta$ 1-42 (Anaspec) or $500 \mu \mathrm{M}$ Glutamic Acid (Sigma) in their regular culture medium. Also, since replacement of conditioned culture medium with fresh medium determines neuronal suffering in $8 \mathrm{~h}$, and complete death in $30 \mathrm{~h}$, medium was changed 16 hours previous to cell damage and viability tests. All compounds were resuspended, when necessary, according to the manufacturer's instructions, and brought to the desired concentration in sterile double distilled water. $A \beta$ 1-42 was solubilized in Hexafluoroisopropanol (HFIP, Sigma) to $200 \mu \mathrm{M}$ to prevent aggregation, and stored at $-80^{\circ} \mathrm{C}$ in aliquots. The amount needed was then thawed, HFIP was evaporated under the cell culture hood, and $A \beta$ resuspended in sterile double distilled water to the desired concentration.

\section{Immunostaining of cultured neurons and transgenic mice brains}

Cells, plated on Poly-D-Lysine coated coverslips (24 well plates), were washed in TBS once and fixed with 4\% PFA for 30' at room temperature (RT), washed again and permeabilized with $0.2 \%$ Triton X-100/TBS for $10^{\prime}$ on ice, and cold methanol for 5 ' on ice. Blocking of aspecific antigenic sites was performed with 10\% Goat Serum/0.2\% Triton X-100/TBS for $1 \mathrm{hr}$ at RT. Primary antibodies were: anti MAP2 (Sigma, monoclonal clone HM-2, 1/500), anti NeuN (Chemicon, monoclonal clone A60, 1/500), anti GFAP (Abcam 7260, polyclonal 1/500). Secondary antibodies were anti-mouse Alexa Fluor 350 and anti rabbit Alexa Fluor 488, all in 5\% Goat Serum/0.1\% Triton X$100 / \mathrm{TBS}$ for 90' at room temperature. All washes in between and after antibodies incubations were $2 \times 10^{\prime}$ with TBS pH7.6/0.2\% Triton X-100. Coverslips were then mouted on Superfrost Plus(+) glass slides with a glycerol based mount and stored at $4^{\circ} \mathrm{C}$, shaded from light. This procedure was optimized in order to obtain maximum reduction of background. Zeiss Axioskop, with fluorescence filters, AxioCam and Axiovision software was used for images acquisition.

\section{Assessment of neuronal toxicity and viability}

Cell suffering was assessed by detecting LDH liberated in the culture medium by damaged neurons treated with toxic/pro-apoptotic stimuli (Roche), according to the manufacturer's instructions. Cell viability was assayed by WST1 incorporation in lively cultured neurons after treatment with toxic/pro-apoptotic stimuli (Roche), according to the manufacturer's instructions.

\section{AID peptide detection and western blots}

Frozen hemispheres were homogenized through sonication ( $3 \times 30 "$ cycles, with 5" pulses) in ice with HU 22575 Sonifier (Branson Sonic Power) at \#4 power. Buffer is as follows: $2 \%$ SDS, $1 \times$ Roche Protease Inhibitors Com-

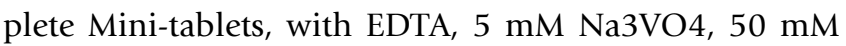
$\mathrm{NaF}, 1 \mathrm{mM}$ DTT, $1 \mathrm{mM}$ PMSF. Homogenates were spun down at $49000 \mathrm{rpm}$ (100000 g) on a TLA110 rotor (Beckman) at $4{ }^{\circ} \mathrm{C}$ for 70'. Supernatants, corresponding to $1 \mathrm{mg}$ of total proteins, quantified using BIORAD Smart Spec 3000 and Protein Assay Reagent, were pre-cleared in Protein A Plus (Pierce) for 4 hours at $4{ }^{\circ} \mathrm{C}$. Lysates were then incubated with $1 \mu \mathrm{g}$ of rabbit C-terminal APP antibody (Zymed) over night at $4^{\circ} \mathrm{C}$. Finally, Protein A Plus (Pierce) was added again and incubated for 4 hours at $4{ }^{\circ} \mathrm{C}$. Beads were washed, resuspended in NuPAGE LDS Sample Buffer/ $\beta$-MercaptoEthanol, boiled, and $10 \mu \mathrm{L}$ were loaded on a $4-12 \%$ NuPAGE gel. Proteins were then blotted on a $0.2 \mu \mathrm{m}$ nitrocellulose membrane (Schleicher \& Schuell), blocked in 5\% milk/PBS and probed with either rabbit anti APP C-terminal antibody (Zymed, 1/500 dilution) or with the rabbit C8 antibody (provided by Dennis Selkoe, $1 / 500$ dilution). Western Blots on homogenates from AIDtg and littermate mice were carried out as described previously [22]. Secondary antibody was a goat anti rabbit-HRP (Southern Biotech, 1/3000 dilution). C8 was diluted in Superblock/PBS (Pierce), while secondary antibody was dilute in 5\% milk/PBS. Blots were developed with SuperSignal West Pico Chemiluminescent Substrate (Pierce) and SuperSignal West Dura Extended Duration Substrate (Pierce).

\section{RT and Quantitative PCR}

Each experiment was done in triplicate. Several primer pairs were tested, prior the experiments, to check for proper amplification and to rule out primer dimerization. Selected primers are as follows:

-hsAID: fw: 5'-GCATCGATTCTAGAATTCG-3'; rev: 5'CCACCACACCATGATGAAT-3'

-hsAPP: fv: 5'-TCGGAAGTGAAGATGGATGC-3'; rev: 5'CCTTTGTTCGAACCCACATC-3'

-mmKAI1: fv: 5'-CCTCTTCCTCTTCAACTTGCT-3'; rev: 5'CGGAAATGAAGCTGTTCTTG-3'

-mmNeprilysin: fw: 5'-GGACATGAAATCACACATGG-3'; rev: 5'-AAATTATTTGCCGACTGCTG-3' 
-mmß-actin: fv: 5'-AAATCGTGCGTGACATCAAA-3; 5'TCTCCAGGGAGGAAGAGGAT-3'.

Mouse brain mRNA was extracted with Trizol reagent (Invitrogen), processed and purified with RNeasy Protect Kit (Qiagen) according to the manufacturers' protocols. Two $\mu \mathrm{g}$ of RNA were retro transcribed to cDNA using SuperScript III First-Strand Synthesis System for RT-PCR kit (Invitrogen). Quantitative PCR was carried out using Power Sybr Green PCR Master Mix on a ABI PRISM 7900 HT Sequence Detection System (Applied Biosystems) according to the manufacturer's protocols. Data analysis was conducted according to M. W. Pfaffl [23] and Applied Biosystems references and protocols.

\section{Sample preparation and hybridization for micro array analysis}

Each experimental point was performed in triplicate. Mouse Brains were homogenized in TRIZOL reagent (Invitrogen) and extracted following the manufacturer's protocol. A further purification step with the PROTECT kit (Qiagen) was added. cRNA was generated by using the Affymetrix One-Cycle Target Labeling and Control Reagent kit (Affymetrix Inc., Santa Clara, California, USA), following the manufacturer's protocol. The biotinylated cRNA was hybridized to the MOE 4302.0 Affymetrix DNA chips, containing over 39000 genes and open reading frames from M. musculus Genome databases GenBank, dbEST and RefSeq. Chips were washed and scanned on the Affymetrix Complete GeneChip ${ }^{\circledast}$ Instrument System, generating digitized image data files.

\section{Micro array data analysis}

DAT files were analyzed by MAS 5.0 for detection calls (Affymetrix Inc.) and RMA for expression values. The expression values obtained were analyzed by using GeneSpring GX (AgilentTechnologies). Results were filtered for flag (presence call), then for fold change $>1.5$, obtaining a total of 5019 probe sets differentially expressed in the samples versus the controls. Statistical analysis was initially performed using the Two-Way ANOVA using Age and Transgene Expression as parameters to test. As Age was the only parameter to give significant results, we next applied a Welch T-Test on Age using as p-value cutoff 0.001 , multiple testing correction Bonferroni, obtaining a set of 380 genes statistically significant. Transgene Expression didn't give any significant result even using a p-value cut-off 0.05 .

\section{Statistical analysis}

All quantified data represent an average of at least triplicate samples. Error bars represent standard errors of the mean. Statistical significance was determined by Student's $\mathrm{t}$ test and a $\mathrm{p}<0.05$ was considered significant.

\section{Results \\ Generation of AID transgenic animals}

To directly examine the effects of AID in vivo, and in the brain, we generated transgenic mice expressing AID under the control of the CaMKII $\alpha$ promoter, targeting its expression to the forebrain regions (which comprise the thalamus, hypothalmus and the upper telencephalon) of the postnatal mouse [24]. These areas are most relevant to Alzheimer's pathology. Endogenous AID is very short lived and therefore virtually undetectable [25]. We generated transgenic lines expressing either the 59- or 57-residue AID peptide, which would be produced by $\gamma$-cleavage together with either $A \beta 40$ or $A \beta 42$, respectively. In addition, transgenic lines expressing a " $\varepsilon$-cleavage" AID of 50residue $[26,27]$ were also made. AID cDNAs were cloned downstream of the $8-\mathrm{Kb}$ CaMKII $\alpha$ promoter and into a plasmid containing a mini-intron and the SV40 polyadenylation sequence [24] (Figure 1A). The linearized plasmids were injected into oocytes of FVB mice that were than implanted in pseudo pregnant C57BL/6 mice. TailDNA PCR, showed that 9 out of 63 pups obtained had integrated the AID transgenes (samples are shown in Figure $1 \mathrm{~B})$. More specifically, we obtained two AID59 (AID59-4.4 and -1.1), four AID57 (AID57-13.3, -5.1, -5.2 and -8.1) and three AID50 (AID50-3.4, -1.5 and 5.2) founder mice. Germline transmission was observed for all founders. The expression levels of the AID transgene mRNA and protein were determined by real-time quantitative PCR and Immunoprecipitation followed by Western blot analysis, respectively. Total RNA and protein lysates were isolated from the forebrain of adult AIDtg animals. Different levels of AID mRNA and AID peptide were found in the different transgenic mice (compare Figure 1C and 1D). Of note, AID50-1.5 and AID50-3.4 lines expressed the highest levels of AID mRNA but no detectable AID50 protein. This data suggests that AID50 is the more unstable AID peptide form.

All mice show, up to 24 months of age, a regular growth pattern and mating ability, and we cannot detect any gross deficit or behavioral abnormality among the different AIDtg lines compared to the wild type littermates.

\section{APP, KAII, NEP and $p 53$ gene expression is not altered in AID transgenic adult animals}

To determine whether AID affects APP, KAI1, NEP and p53 mRNA expression in vivo in the brain, RNA from the forebrain of adult (3-8 months) AID57-5.1, AID57-13.3, AID59-4.4, AID59-1.1, AID50-1.5, AID50-3.4, AID50-5.2 and control littermates were analyzed by real-time quantitative PCR. The data presented in Figure 2 show that there is no obvious correlation between AID mRNA and $A P P, K A I 1, N E P$ and p53 levels, considering also age, sex and AID transgene levels of expression. Overall, these data suggest that AID is not involved in the basal expression of 


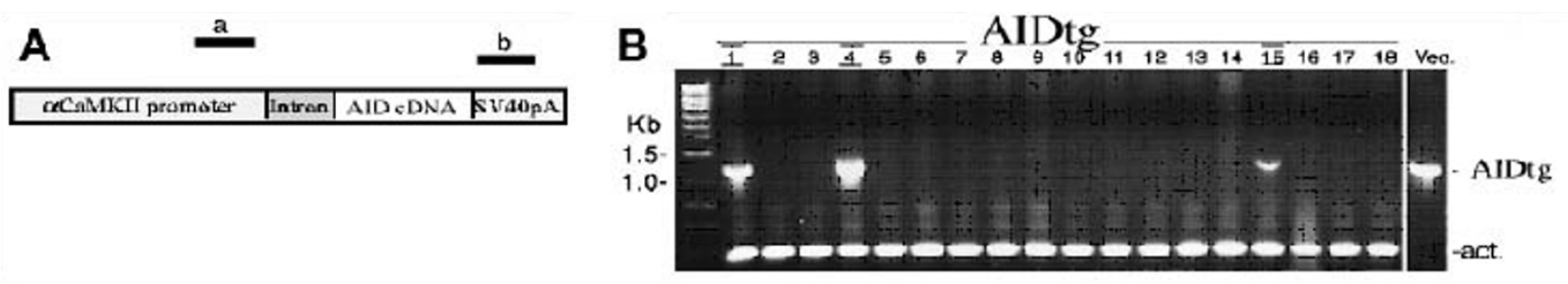

C

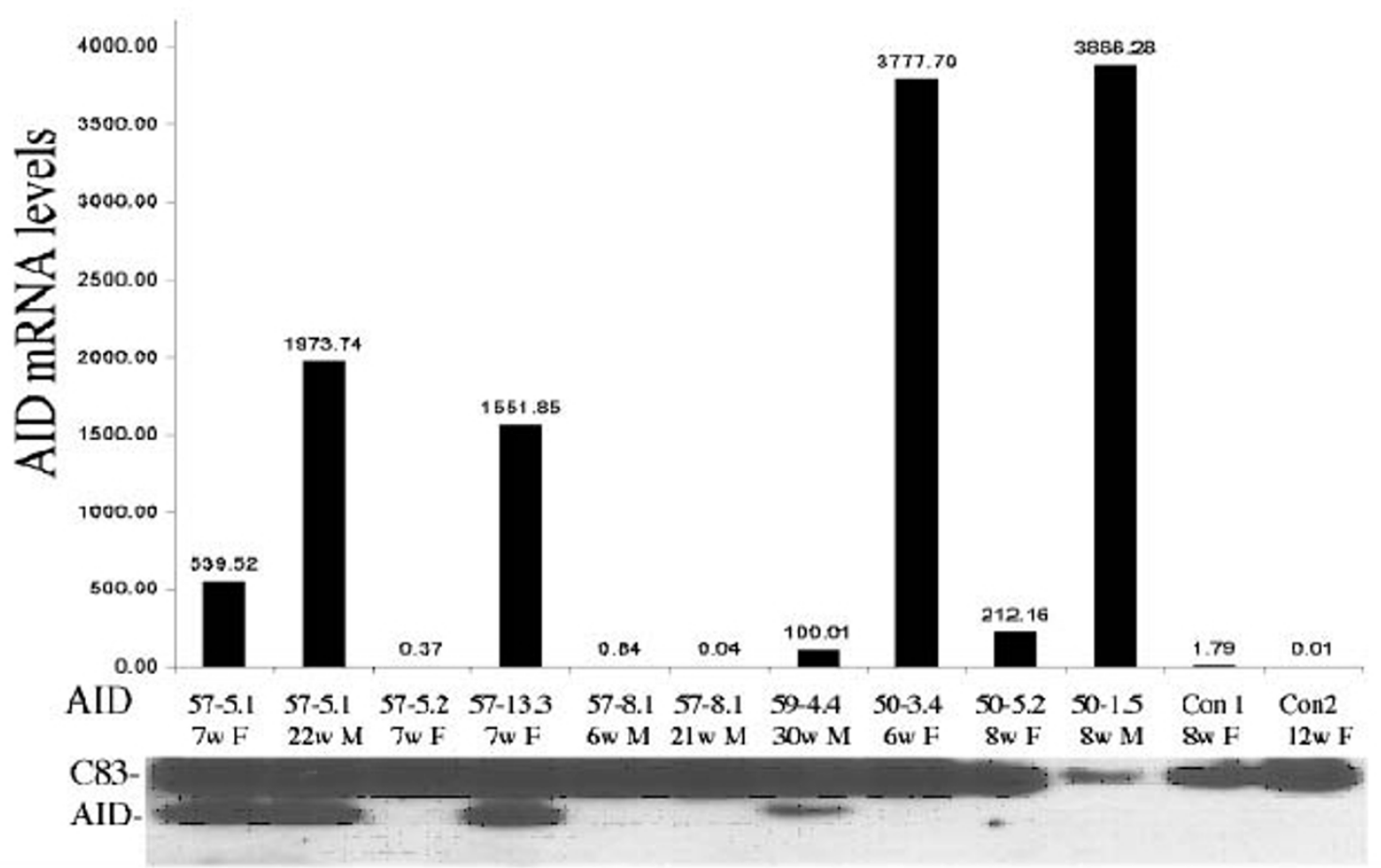

Figure I

Characterization of AID transgenic mice. A Schematic representation of the transgenic AID constructs (fragments are not depicted in scale). The location of the PCR primers $a$ and b used for genotyping is shown. B PCR of 63 pups (I8 are shown here) revealed that 9 of them (three are shown here, number I, 4 and I5) had integrated the AID transgene. In the same PCR tube, $\beta$-actin was amplified to control for genomic DNA content. Vec. represents the control PCR performed using the transgenic vector as a template. C Real Time PCR showing the expression levels of the AID transgene in different lines. D Immunoprecipitation and western blot was conducted from a brain hemisphere of AIDtg and littermate (Con) mice. Mice lines that expressed the AID protein at the mRNA level, and had a detectable band at the western blot analysis were selected for further studies.

putative AID transcriptional targets in the adult mouse forebrain.

\section{AID does not regulate basal gene expression in the mouse brain}

A role for AID in transcription cannot be nonetheless excluded from the above evidence. AID might indeed regulate transcription of yet unidentified genes. To address this point we took advantage of our AID tg mice. It is foreseeable that an AID target should be dis-regulated in the forebrain of AID $\mathrm{tg}$ mice. RNA from the forebrain of AID59-4.4, AID57-5.1, AID57-13.3 and control litterma- tes was prepared from either 9 days or 18 days old mice. AID50tg mice were not included in this analysis because we could not detect expression of the AID50 peptide. Nine day old animals were selected as further negative controls because the transgenic cassette should be expressed only two weeks after birth. However, we detected expression of AID mRNA before day 18, in the 9 days old AIDtg (Figure 3). Before using these samples for micro array analysis, the RNAs were tested for APP, KAI1, NEP and $p 53$ expression. Once more, we saw no clear-cut correlation between expression of AID and that of its putative gene targets (Figure 3, AID 57-5.1 and 13.3 

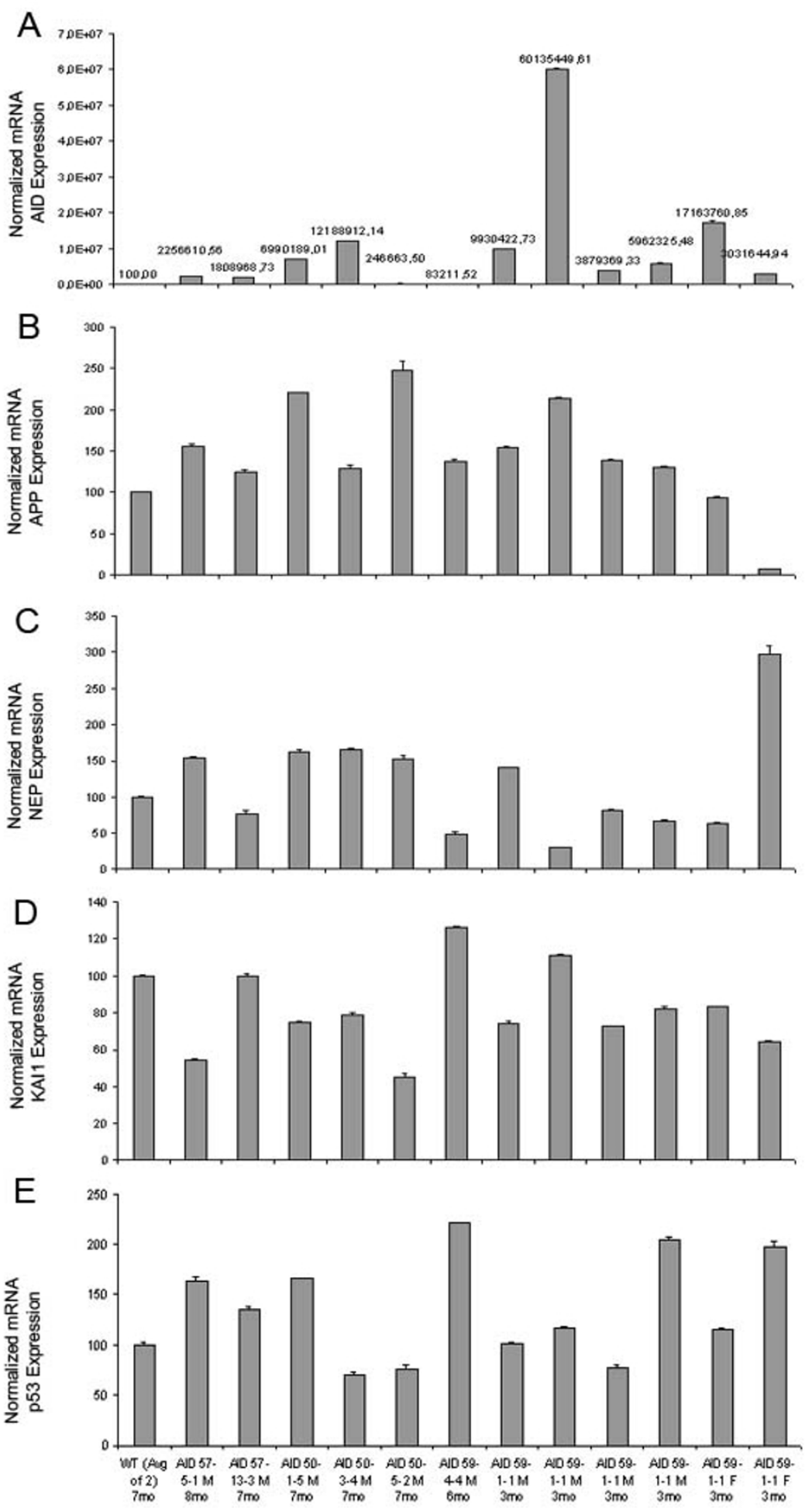

Figure 2

In vivo expression of candidate AID targets is not affected by transgenic AID expression, in adult mice. Real Time Quantitative PCR shows the relative expression of AIDtg protein, A, APP, B, NEP, C, KAII, D, and p53, E in the forebrain and hippocampus of AIDtg and their littermate control mice. Values are relative to $100 \%$ value given arbitrarily to a littermate mouse. Experiments were conducted in triplicate loading, and the error bars represent standard deviations. 

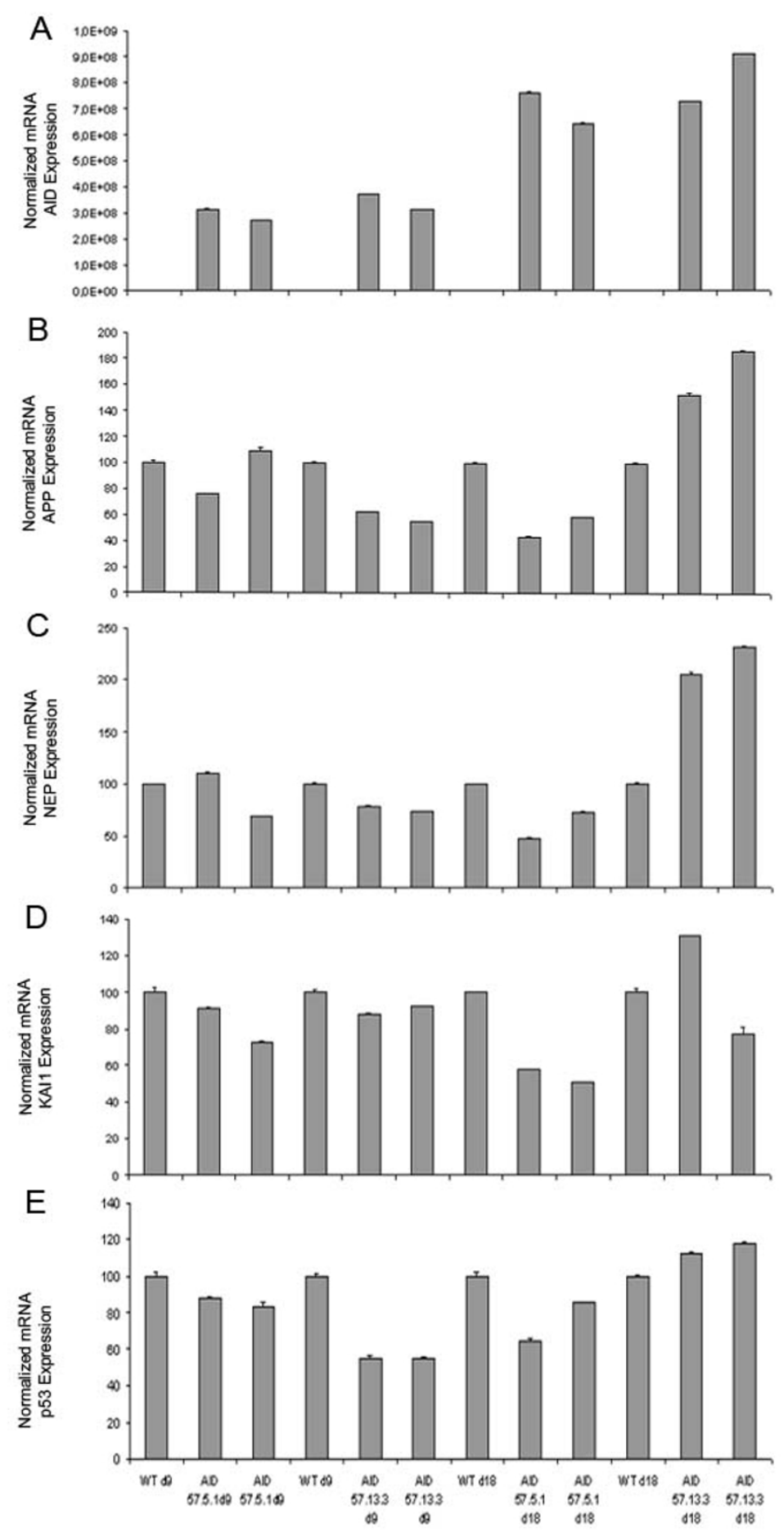

Figure 3

AID is already expressed at postnatal day nine, but does not influence the expression of the candidate genes. Real Time Quantitative PCR shows the relative expression of AIDtg protein A, APP, B, NEP, C, KAII, D, and p53, E in the forebrain and hippocampus of AIDtg and their littermate control mice as early as 9 and 18 days post-natal. For APP, NEP, KAII and p53 expression, values are relative to $100 \%$ value given arbitrarily their day 9 and day 18 littermates. For AIDtg protein, $100 \%$ value was assigned to the first day 9 littermate mouse. Experiments were conducted in triplicate loading, and the error bars represent standard deviations. 
shown). Since we did not test EGFR and LRP mRNA levels, those genes (even their basal transcription) may still be regulated by AID alone, without over-expression of Fe65. Regardless, we analyzed the forebrain transcriptome of these mice using an Affymetrix DNA chips, containing over 39000 genes and open reading frames from M. musculus Genome databases GenBank, dbEST and RefSeq. Statistical analysis performed using age and transgene expression as parameters to test, showed that age difference was the only parameter to give significant results, yielding a set of 380 genes that were differentially expressed between 9 and 18 day old mice (data not shown), indicating changes in the transcriptome during post-natal development. Transgene expression didn't give any significant result even using a p-value cut-off 0.05 indicating that the forebrain transcriptome was identical in all age-matched mice analyzed. The above data argue against a role for AID in basal transcriptional regulation.

\section{Transgenic AID expression is detectable in fetal neurons in culture, does not influence the expression of target genes, but increases neuronal sensitivity to toxic and apoptotic stimuli}

The finding that TgAID, under the control of our forebrain promoter, is expressed even at postnatal day 9, has led us to think that we could exploit the potentiality of neuronal cultures to assess the role of AID. Neurons from embryonic day 17.5 fetuses, cultured for 9 days, showed detectable TgAID expression (Figure 4A). As for postnatal and adult mice though, the presence of AID 57 (data not shown for AID 59) did not seem to influence the relative expression of our target genes (Figure 4B-E).

Since AID has been implicated in pathways leading to cell death and apoptosis $[3,12,28]$ we aimed to determine its role under cellular stress conditions. We prepared neuronal cultures from AID59 (and AID57, not shown) mice and littermates. Purity of these cultures was assessed by staining for Microtubule Associated protein 2 (MAP2), Neuronal Nuclei (NeuN) and Glial fibrillary Acidic Protein (GFAP) (Figure 5A and 5B). MAP2 is the major microtubule associated protein of brain tissue, is known to promote microtubule assembly and to form side-arms on microtubules. It also interacts with neurofilaments, actin, and other elements of the cytoskeleton. It electively stains dendrites. NeuN (or Neuronal Nuclei) reacts with most neuronal cell types throughout the nervous system of mice including cerebellum, cerebral cortex, hippocampus, thalamus. Developmentally, immunoreactivity is first observed shortly after neurons have become postmitotic. The immunohistochemical staining is primarily localized in the nucleus of the neurons. GFAP is a member of the class III intermediate filament protein family. It is heavily, and specifically, expressed in astrocytes and certain other astroglia in the central nervous system. Antibodies to
GFAP are therefore very useful as markers of astrocytic cells.

As shown in figure 5C, AID59 (analogous results for AID57, not shown) positive neurons have a lower threshold to cell damage induced by toxic or pro-apoptotic stimuli, as indicated by LDH release in culture medium. In particular, H2O2, Kainate and Staurosporine show the biggest differences in LDH release between AID positive and negative cells; the biggest difference in cell viability is seen in Kainate and Staurosporine treatments. When $1 \mu \mathrm{M}$ A $\beta$ 1-42, $500 \mu \mathrm{M}$ Glutamate and "starvation" were used as noxious stimuli, no difference was detected between transgenic and non-transgenic neurons (data not shown). As expected, there was no difference in these indicators, between AID positive and negative neurons, in nontreated cells (not shown). In figure 5D, LDH release was weighed with WST-1 incorporation in the same cells. It is possible that transcription of genes involved in apoptosis, e.g., p53 etc, may be regulated by AID under the stress or pathological conditions.

\section{Discussion and Conclusion}

The findings that AID might regulate apoptosis and $\mathrm{Ca++}$ flux were met with some skepticism. On the contrary, hints to a transcriptional role of AID generated great enthusiasm given the parallel with Notch signaling. Several reports have pointed to few possible AID transcriptional targets. The evidence that AID is found on the KAI1 promoter, where it is perhaps complexed to $\mathrm{Fe} 65$ and the hystone acetyltransferase Tip60 [9], have supported a direct role for AID in transcription. It can also be hypothesized that AID regulates transcription indirectly and that APP functions as a "hanger" that restrains Fe65 outside the nucleus: APP processing would activate transcription, as it liberates Fe65 and allows it to translocate to the nucleus. More recent data have suggested that the APP/ Fe65 interaction promotes a "conformational maturation" of Fe65 that is converted into a transcriptionally active state [29]. However, the reported AID-dependent changes in gene expression has been questioned [18]. Therefore, we have reexamined the role of AID in in vivo transcription. Overexpression of AID in the mouse brain did not affect the levels of these three putative AID gene targets. Although these findings question the role of AID in basal transcription of these candidate genes, it is still possible that authentic AID targets genes have not been yet characterized. To address this we have analyzed the effect of AID expression on the mouse brain transcriptoma. The data show that the gene expression pattern of AIDtg and littermate mice is identical, failing to identify any other potential AID gene targets. Thus, AID might have a transcriptional function either in a small subset of forebrain neuronal cells, in cell types different than those analyzed here or under specific signaling or stressful con- 

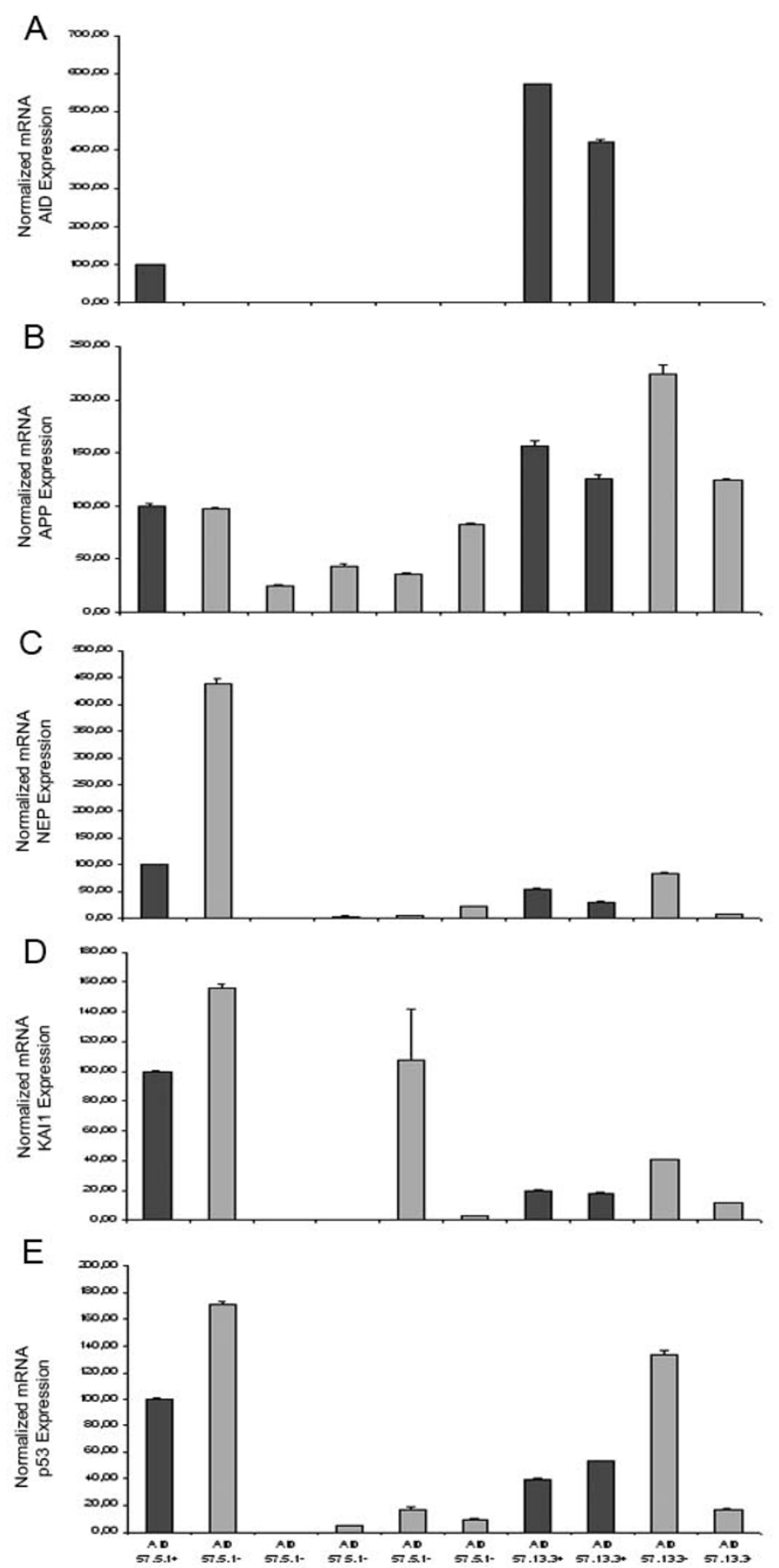

Figure 4

AIDtg expression in cultured fetal primary neurons does not change the relative expression of $A P P, N E P, K A I I$ and p53. A AIDtg expression was confirmed by tail genotyping of fetuses (not shown) and by QPCR data on cultured neurons (dark bars). Expression of AID, A, APP, B, NEP, C, KAII, D, and $p 53$, E, is relative to the $100 \%$ value given arbitrarily to the first AIDtg mouse. Experiments were conducted in triplicate loading, and the error bars represent standard deviations. Cultures were harvested at DIV I4. Similar results were achieved from younger cultures (DIV 9, not shown) and in the AIDTg 59 line (not shown). 
A

$\mathrm{B}$
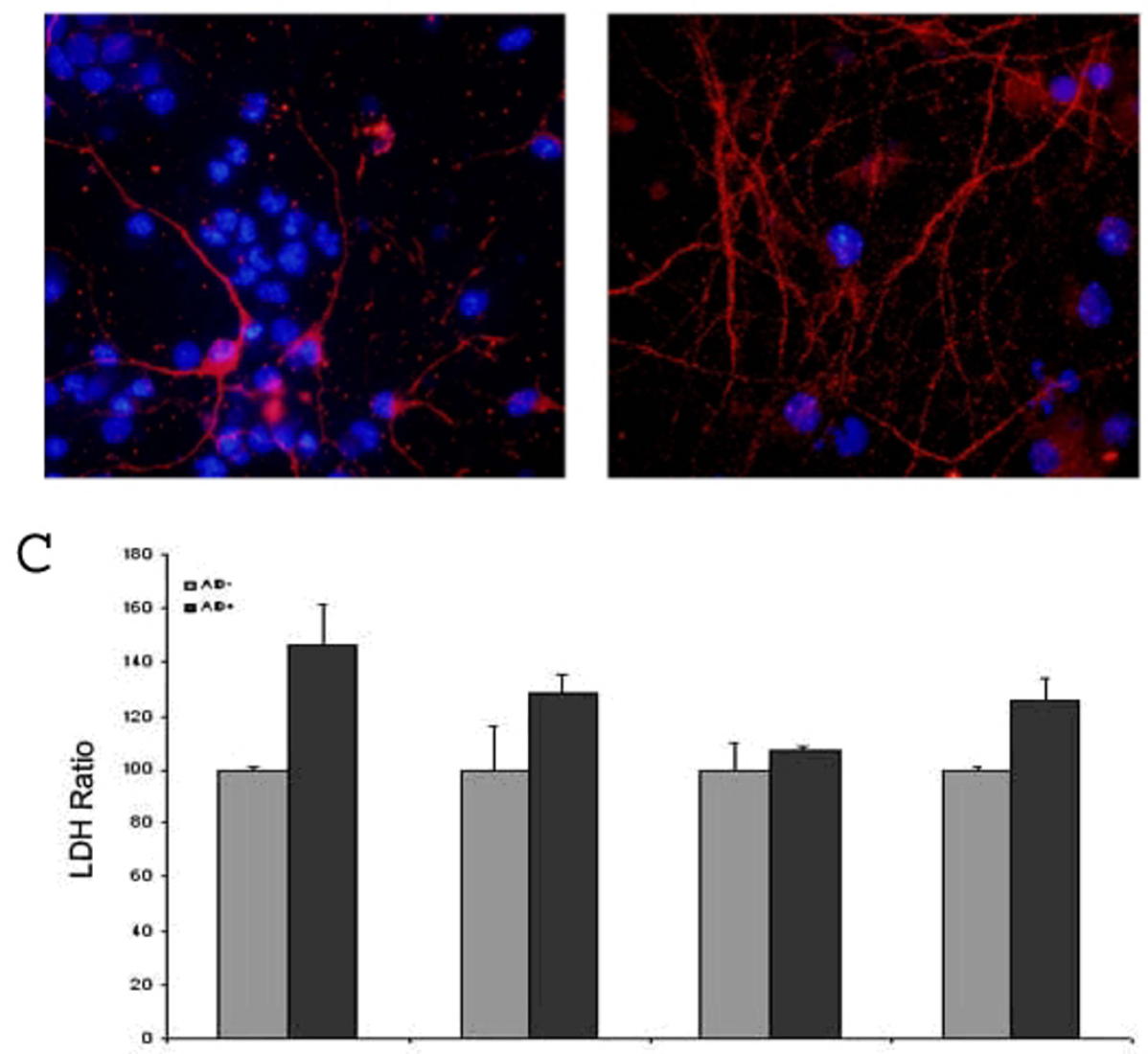

$\mathrm{D}$

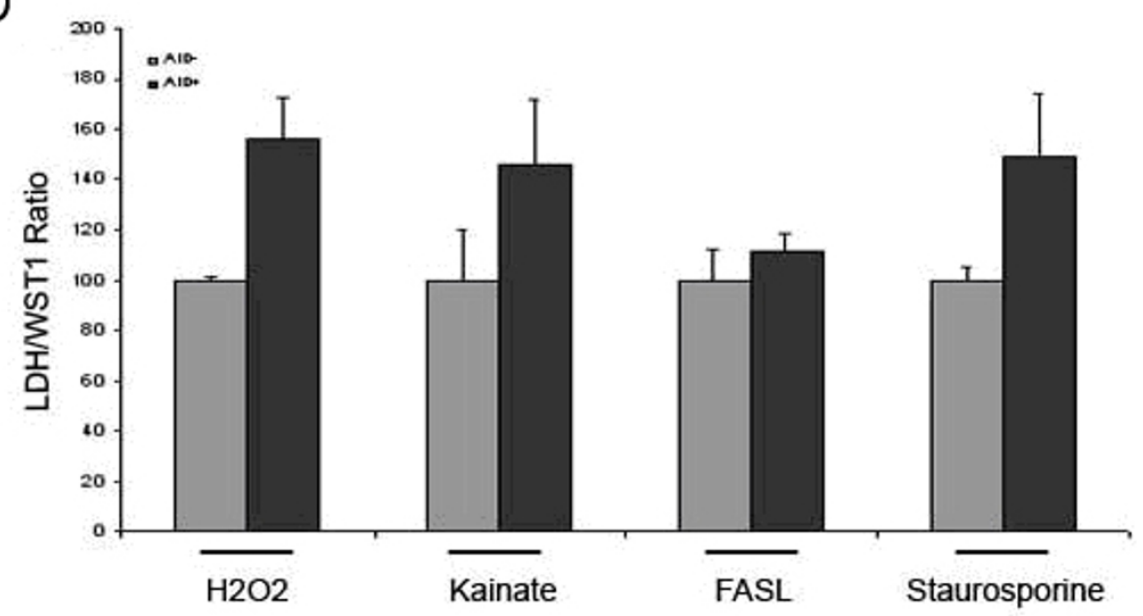

Figure 5

AIDtg expression in cultured fetal primary neurons increases their sensitivity to toxic and apoptotic stimuli. AIDTg line 59.4.4, at DIV 9. To verify the purity of the neuronal cultures, cells were stained with anti-NeuN (Blue) plus antiGFAP (red), A, and NeuN (Blue) plus anti-MAP2 (red), B. C Toxicity of indicated stimulus was assessed by measuring LDH release. D Released LDH was weighed against WST-I uptake in the LDH/WST-I ratio, which confirms the trend of LDH release. $\mathbf{C}$ and $\mathbf{D}$ Values from toxic stimuli were weighed against values from untreated cells to express the increase of the indicators of cell damage. Average values refer to at least 3 AIDtg and 3 littermate mice; measurements were done on 6 separate wells of 96 well culture plate for each foetus' neurons. Similar results were obtained for AIDTg 57 line (not shown). 
ditions. Genome-wide analysis, conducted on neuronal cells expressing inducible AID, has shown that several genes involved in cytoskeletal dynamics can be regulated by AID. The finding has been confirmed, by SYBR Green real-time PCR, in brains of $\mathrm{AD}$ patients for 2-Actin, IGFBP3, and TAGLN [17]. These target genes do not seem to be regulated by AID in our model. This might be due to two reasons. Induction of the AID transgene was allowed for 72 hours in culture before any effect could be detected. Our mice overexpressed AID for several days, as also evident by AID mRNA detection in cultured neurons. It is foreseeable that any effect of AID overexpression, during a longer period of time and in a more complex setting, as is the living mouse brain, would probably result in a different expression arrangement, especially of genes devoted to maintaining the integrity of the cell. Also, sporadic $\mathrm{AD}$ brains are a much more complex and entropic system than ours, allowing for complex interactions between different pathogenic entities. Thus, we cannot exclude that a dis-regulation of these target genes may happen later in the life of our mice or under different stress conditions. The role of the intracellular fragment of APP, could possibly be understood by studying its effect under specific stress situations, e.g. under apoptotic or oxidative stimuli, where it could play either a protective or a detrimental role for the cell, depending on other factors such as cell types and interaction with other proteins. This would also explain the predisposition of some brain regions to Alzheimer's pathology. AID has been proposed as a possible mediator of cell death, via a reduction of the cellular threshold to apoptosis [3]. But recent findings have also pointed to a possible protective effect of the APP c-terminal/Fe65 interaction, involving DNA damage response [28]. Our data show that over-expression of AID in cultured neuronal cells predisposes them to a higher degree of suffering, i.e. to a lower resistance to toxic and apoptotic stimuli. In our hands, only selective stimuli could reveal this peculiarity, possibly because of different threshold to cell damage for each experimental compound. Recent works show a role for AID in p53 associated cell death [10]. In our model, under toxic stimuli, AID may lower the threshold to cell death through a p53dependent mechanism by augmenting p53 expression. However, further experiments are required to test this hypothesis.

We believe that the key to understand the role of APP processing in gene regulation lays in the complex interaction of APP domains with other intra- or extra-cellular factors, possibly having a role only in certain stressful situation or at a given "age". Further work will explore the nature of this complex network.

\section{Competing interests}

The authors declare that they have no competing interests.

\section{Authors' contributions}

LG participated in the design of the study, handled the mice colony and genotyping, designed the experiments, performed most of the experiments and cultures, participated in the final analysis and draft preparation. DZ participated in the mice colony handling and genotyping, and characterization of $\mathrm{tg}$ mice. RW participated in the mice colony handling and genotyping. ET participated in the design of the study. PDL performed all the micro-array experiments and analysis. MT participated in the design of the study. LD conceived and designed the study, designed the tg mice, participated in the design of the experiments, participated in the handling of the mice colonies and genotyping, and in the analysis of the data, prepared the draft.

\section{Acknowledgements}

This work was supported in part by Alzheimer Disease Research Grant A2003-076; National Institutes of Health Grants ROI AG22024 and ROI AG2 I588, the CARISA Foundation, the MIUR and the Regione Piemonte.

\section{References}

I. Zheng $\mathrm{H}$, Koo $\mathrm{EH}$ : The amyloid precursor protein: beyond amyloid. Mol Neurodegener 2006/08/26 edition. 2006, I:5.

2. Selkoe DJ, Podlisny MB: Deciphering the genetic basis of Alzheimer's disease. Annu Rev Genomics Hum Genet 2002, 3:67-99.

3. Passer B, Pellegrini L, Russo C, Siegel RM, Lenardo MJ, Schettini G, Bachmann M, Tabaton M, D'Adamio L: Generation of an apoptotic intracellular peptide by gamma-secretase cleavage of Alzheimer's amyloid beta protein precursor. J Alzheimers Dis 2000, 2(3-4):289-30I.

4. Leissring MA, Murphy MP, Mead TR, Akbari Y, Sugarman MC, Jannatipour M, Anliker B, Muller U, Saftig P, De Strooper B, Wolfe MS, Golde TE, LaFerla FM: A physiologic signaling role for the gamma -secretase-derived intracellular fragment of APP. Proc Natl Acad Sci U S A 2002/03/28 edition. 2002, 99(7):4697-4702.

5. Cao X, Sudhof TC: A transcriptionally [correction of transcriptively] active complex of APP with Fe65 and histone acetyltransferase Tip60. Science 200I, 293(5527): I I5-I 20.

6. Artavanis-Tsakonas S, Rand MD, Lake RJ: Notch signaling: cell fate control and signal integration in development. Science 1999/ 04/30 edition. 1999, 284(54 I 5):770-776.

7. Annaert W, De Strooper B: Presenilins: molecular switches between proteolysis and signal transduction. Trends Neurosci 1999/09/I I edition. 1999, 22(10):439-443.

8. Wiley JC, Smith EA, Hudson MP, Ladiges WC, Bothwell M: Fe65 stimulates proteolytic liberation of the beta-amyloid precursor protein intracellular domain. J Biol Chem 2007/09/15 edition. 2007, 282(46):333।3-33325.

9. Baek SH, Ohgi KA, Rose DW, Koo EH, Glass CK, Rosenfeld MG: Exchange of N-CoR corepressor and Tip60 coactivator complexes links gene expression by NF-kappaB and beta-amyloid precursor protein. Cell 2002/08/02 edition. 2002, I | 0( I):55-67.

10. Pardossi-Piquard R, Petit A, Kawarai T, Sunyach C, Alves da Costa C, Vincent B, Ring S, D'Adamio L, Shen J, Muller U, St George Hyslop P, Checler F: Presenilin-dependent transcriptional control of the Abeta-degrading enzyme neprilysin by intracellular domains of betaAPP and APLP. Neuron 2005, 46(4):54I-554.

II. Ryan KA, Pimplikar SW: Activation of GSK-3 and phosphorylation of CRMP2 in transgenic mice expressing APP intracellular domain. J Cell Biol 2005/I0/19 edition. 2005, I 7 I(2):327-335.

12. Kim HS, Kim EM, Lee JP, Park CH, Kim S, Seo JH, Chang KA, Yu E, Jeong SJ, Chong YH, Suh YH: C-terminal fragments of amyloid precursor protein exert neurotoxicity by inducing glycogen synthase kinase-3beta expression. Faseb J 2003/08/19 edition. 2003, I7(13): 1951-1953.

13. Zhang YW, Wang R, Liu Q, Zhang H, Liao FF, Xu H: Presenilin/ gamma-secretase-dependent processing of beta-amyloid 
precursor protein regulates EGF receptor expression. Proc Natl Acad Sci U S A 2007/06/09 edition. 2007, 104(25): 10613-106I8.

14. Liu Q, Zerbinatti CV, Zhang J, Hoe HS, Wang B, Cole SL, Herz J, Muglia L, Bu G: Amyloid precursor protein regulates brain apolipoprotein $\mathrm{E}$ and cholesterol metabolism through lipoprotein receptor LRPI. Neuron 2007/10/09 edition. 2007, 56(I):66-78

15. von Rotz RC, Kohli BM, Bosset J, Meier M, Suzuki T, Nitsch RM, Konietzko U: The APP intracellular domain forms nuclear multiprotein complexes and regulates the transcription of its own precursor. J Cell Sci 2004, I 17 (Pt 19):4435-4448.

16. Ahn KW, Joo Y, Choi Y, Kim M, Lee SH, Cha SH, Suh YH, Kim HS: Swedish amyloid precursor protein mutation increases cell cycle-related proteins in vitro and in vivo. J Neurosci Res 2008/ 04/29 edition. 2008, 86( I I):2476-2487.

17. Muller T, Concannon CG, Ward MW, Walsh CM, Tirniceriu AL, Tribl $F$, Kogel D, Prehn JH, Egensperger R: Modulation of gene expression and cytoskeletal dynamics by the amyloid precursor protein intracellular domain (AICD). Mol Biol Cell 2007, 18(I):20I-210.

18. Hebert SS, Serneels L, Tolia A, Craessaerts K, Derks C, Filippov MA, Muller $U$, De Strooper B: Regulated intramembrane proteolysis of amyloid precursor protein and regulation of expression of putative target genes. EMBO Rep 2006/05/27 edition. 2006, 7(7):739-745.

19. Waldron E, Isbert S, Kern A, Jaeger S, Martin AM, Hebert SS, Behl C, Weggen S, De Strooper B, Pietrzik CU: Increased AICD generation does not result in increased nuclear translocation or activation of target gene transcription. Exp Cell Res 2008/06/19 edition. 2008, 3 I 4(I3):24|9-2433.

20. Ozaki T, Li Y, Kikuchi H, Tomita T, Iwatsubo T, Nakagawara A: The intracellular domain of the amyloid precursor protein (AICD) enhances the p53-mediated apoptosis. Biochem Biophys Res Commun 2006, 35I(I):57-63.

21. Checler F, Sunyach C, Pardossi-Piquard R, Sevalle J, Vincent B, Kawarai T, Girardot N, St George-Hyslop P, da Costa CA: The gamma/epsilon-Secretase-Derived APP Intracellular Domain Fragments Regulate p53. Curr Alzheimer Res 2007, 4(4):423-426.

22. Scheinfeld MH, Roncarati R, Vito P, Lopez PA, Abdallah M, D'Adamio L: Jun NH2-terminal kinase (JNK) interacting protein I (JIPI) binds the cytoplasmic domain of the Alzheimer's beta-amyloid precursor protein (APP). J Biol Chem 2002, 277(5):3767-3775.

23. Pfaffl MW: A new mathematical model for relative quantification in real-time RT-PCR. Nucleic Acids Res 2001/05/09 edition. 200I, 29(9): e45.

24. Abel T, Nguyen PV, Barad M, Deuel TA, Kandel ER, Bourtchouladze $R$ : Genetic demonstration of a role for PKA in the late phase of LTP and in hippocampus-based long-term memory. Cell 1997/03/07 edition. 1997, 88(5):615-626.

25. Cupers P, Orlans I, Craessaerts K, Annaert W, De Strooper B: The amyloid precursor protein (APP)-cytoplasmic fragment generated by gamma-secretase is rapidly degraded but distributes partially in a nuclear fraction of neurones in culture. $J$ Neurochem 200I, 78(5): I |68-II78.

26. Yu C, Kim SH, Ikeuchi T, Xu H, Gasparini L, Wang R, Sisodia SS: Characterization of a presenilin-mediated amyloid precursor protein carboxyl-terminal fragment gamma. Evidence for distinct mechanisms involved in gamma -secretase processing of the APP and Notch I transmembrane domains. J Biol Chem 200I/I0/05 edition. 200I, 276(47):43756-43760.

27. Gu Y, Misonou H, Sato T, Dohmae N, Takio K, Ihara Y: Distinct intramembrane cleavage of the beta-amyloid precursor protein family resembling gamma-secretase-like cleavage of Notch. J Biol Chem 200I/08/03 edition. 200I, 276(38):35235-35238.

28. Minopoli G, Stante M, Napolitano F, Telese F, Aloia L, De Felice M, Di Lauro R, Pacelli R, Brunetti A, Zambrano N, Russo T: Essential roles for Fe65, Alzheimer amyloid precursor-binding protein, in the cellular response to DNA damage. J Biol Chem 2006/I I/24 edition. 2007, 282(2):83I-835.

29. Cao X, Sudhof TC: Dissection of amyloid-beta precursor protein-dependent transcriptional transactivation. I Biol Chem 2004/03/27 edition. 2004, 279(23):2460I-246II. http.//www.molecularneurodegeneration.com/content/3/1/12 